\title{
UV-LED LITHOGRAPHY FOR 3-D HIGH ASPECT RATIO MICROSTRUCTURE PATTERNING
}

\author{
Jungkwun 'JK' Kim*, Seung-Joon Paik, Florian Herrault, Mark G. Allen
}

School of Electrical and Computer Engineering, Georgia Institute of Technology, Atlanta, GA, USA

\begin{abstract}
This paper presents a UV lithography method that utilizes a UV-LED (ultraviolet light-emitting diode) array as a micropatterning light source for high aspect ratio polymer microstructure fabrication. The sidewalls of commercial 5-mm-diamater UV-LEDs were coated with an opaque polymer to suppress the side propagation of UV light and to enhance UV collimation, resulting in a maximum flare angle of $15^{\circ}$. The UV-LEDs were then assembled into a 10x10 array to form the light source. Although the flare angle of most conventional UV lithography tools is $2 \sim 3^{\circ}$, resulting in a slightly better light collimation quality, the polymer structures microfabricated with the UV-LED approach showed similar reproduction fidelity and similar exposure times to patterns fabricated using conventional lithography. The UV-LED approach has the potential for cost reduction as well as simplicity of initial system setup and management. This latter feature is of particular importance in advanced, inclined three-dimensional (3-D) lithography schemes to create complex 3-D structures. The UV-LED system has been demonstrated to perform satisfactorily in these advanced 3-D lithography schemes.
\end{abstract}

\section{INTRODUCTION}

UV lithography systems based on mercury lamp sources have traditionally been used in micro/nano patterning [1]. Modifications of these basic systems, such as deep UV lithography [2] and addition of UV filters [3], can further enhance fine resolution patterning. In MEMS, mercury-lamp-based UV lithography has been further extended to encompass unconventional methods including thermal reflow processes [4], multidirectional UV lithography [5], diffuser lithography [6], and timed-development and-thermal-reflow process [7].

Recent advances in light emitting diode technology have resulted in LEDs that emit in the near ultraviolet. Such UV-LEDs have been used not only in conventional applications such as counterfeit detection (bills, credit cards, documents, etc.) and sterilization, but also as a microfabrication tool. Examples include a

Table1: Comparison between UV-LED[10] and Mercury lamp[11].

\begin{tabular}{|l|l|l|}
\hline & $\begin{array}{l}\text { UV LED (100 } \\
\text { LEDs, } \\
\text { RL5-UV0315-380) }\end{array}$ & $\begin{array}{l}\text { Mercury lamp } \\
(6286 \text { Mercury } \\
\text { Arc Lamp) }\end{array}$ \\
\hline Lifetime & $\begin{array}{l}\text { Approximately } \\
2,000 \text { hours @ } \\
30 \mathrm{~mA} / 23^{\circ} \mathrm{C}\end{array}$ & $\begin{array}{l}\text { Approximately } \\
1,000 \text { hours @ } \\
6 \mathrm{~A}\end{array}$ \\
\hline Radiation Power & $\begin{array}{l}8 \mathrm{~mW} / \mathrm{cm}^{2} @ 12 \mathrm{~W} \\
(30 \mathrm{~mW} / \mathrm{LED})\end{array}$ & $\begin{array}{l}20 \mathrm{~mW} / \mathrm{cm}^{2} @ \\
350 \mathrm{~W}\end{array}$ \\
\hline $\begin{array}{l}\text { Wavelength } \\
\text { characteristics }\end{array}$ & $\begin{array}{l}\text { Single peak (380 } \\
\mathrm{nm})\end{array}$ & $\begin{array}{l}\text { Multiple peaks } \\
(365 \mathrm{~nm}, 405 \\
\mathrm{nm}, 436 \mathrm{~nm})\end{array}$ \\
\hline $\begin{array}{l}\text { Ancillary systems } \\
\text { (power supply, } \\
\text { mirrors, etc.) }\end{array}$ & Simple & Complex \\
\hline
\end{tabular}

single UV-LED for direct write lithography [8], and an array of LEDs in which the spatially nonuniform emission of the LEDs was exploited for microlens array fabrication [9]. Since the UV-LED has the advantages of simple operation and relatively low cost, yet produces high quality near-monochromatic light, it shows great potential for use in microfabrication. Table 1 compares the performance of UV-LEDs with a mercury lamp for several key parameters of interest for UV light source designers. It can be seen that the UV-LED shows performance very comparable to the conventional light source.

In this study, the efficacy of a 100-element array of modified UV-LEDs as a light source for conventional and advanced (3-D) UV lithography is assessed. The modification consisted of suppressing the high flare angle light of each UV-LED by means of an opaque blocking layer. The intensity distribution and attenuation as a function of distance of the array are measured. The performance of the UV-LED source is also compared to the performance of a conventional mercury-lamp-based lithography system.

\section{SYSTEM SET-UP AND TEST}

A 5mm through hole LED (RL5-UV0315-380, Superbrightleds, Inc) is adopted for the UV-LED exposure system. This ultraviolet LED has a single wavelength peak centered at $380 \mathrm{~nm}$ with a maximum radiant power of $30 \mathrm{~mW}$. It has a clear epoxy lens situated on top of a transparent cylindrical package, resulting in a viewing angle of 15 degrees. In order to suppress the significant light leakage through the sidewall of the cylindrical package, the sidewall was coated with an opaque epoxy band to block any UV light emitted through the sidewall.

Figure 1 shows a notional ray trace of light emitted from the LED and traversing to a photoresist-coated mask substrate. The photoresist (e.g., SU-8) in Figure 1 is illuminated using backside exposure (although note that the UV-LED technique is not restricted to backside exposure). After the UV light emerges from the LED lens, it has a $15^{\circ}$ viewing angle, which is equivalent to a $7.5^{\circ}$ half-flare angle incident upon the glass photomask. Application of Snell's law results in a final refracted angle at the photoresist layer

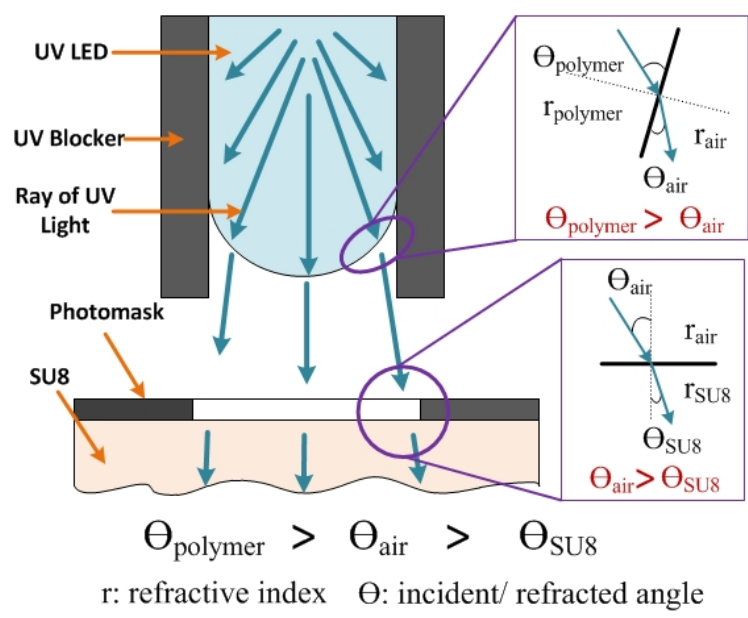

Figure 1: Schematic diagram of UV-LED lithography.

Solid-State Sensors, Actuators, and Microsystems Workshop Hilton Head Island, South Carolina, June 3-7, 2012 


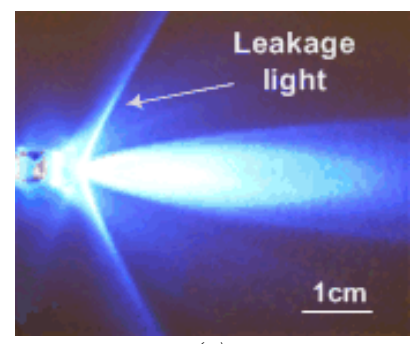

(a)

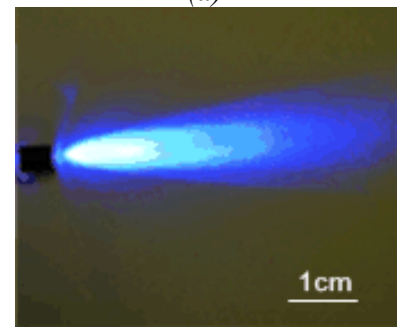

(c)

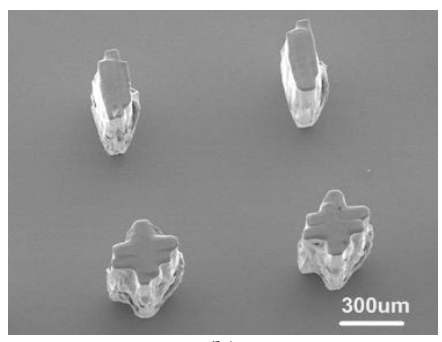

(b)

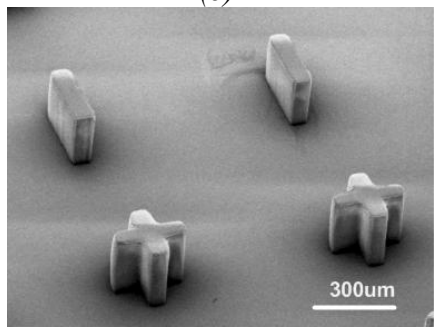

(d)
Figure 2: UV-LED light propagation with and without LED sidewall coating: (a) UV-LED Light emitting without coating, (b) SU-8 patterning with normal LED source, (c) SU-8 pattering with $U V-L E D$ light without sidewall coating, (d) SU-8 patterning with UV-blocking sidewall coating.

of $4.43^{\circ}$ where the refractive indices of air, glass, and SU- 8 are assumed as 1, 1.3, and 1.69 respectively [5]. Since the SU-8 exhibits generally a negatively sloped sidewall (undercut) in top exposure and vice versa in backside exposure caused by diffraction and attenuation, the $4.43^{\circ}$ of the refracted angle enhance the sidewall profile.

Figure 2 shows the profile of the emitted light from a single UV-LED with and without coating the sidewall with opaque material (left), as well as typical SU-8 exposure and development results from each LED (right). The emitted light from a single LED is mostly propagated in the forward direction through the top mounted clear lens. However, there is a considerable amount of leakage light as shown in Figure 2(a). Leakage light results in non-uniform sidewalls and multiple patterning of the fabricated microstructures as shown in Figure 2(b). Figure 2(c) shows the UV-LED with opaque sidewalls and suppressed leakage. As illustrated in Figure 2(d), the corresponding fabricated microstructures exhibit straight sidewalls.

To expose larger areas, a 10x10 element LED array is constructed. This array is realized on a custom printed circuit board (PCB) where the $30 \mathrm{~m}$ thick double sided copper layers were

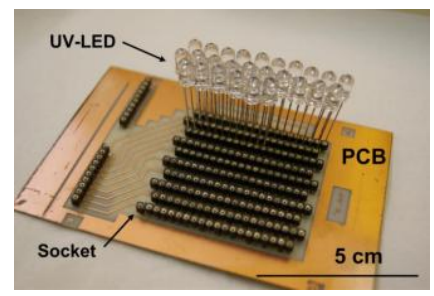

(a)

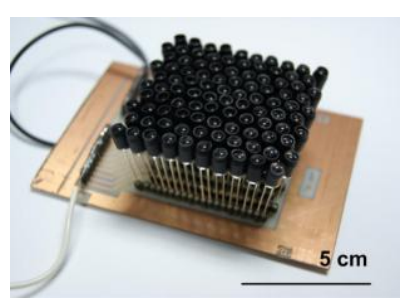

(b)
Figure 3: UV-LED system overview: (a) Circuit board bearing IC socket and UV-LEDs, (b) Completed UV-LED array light source.

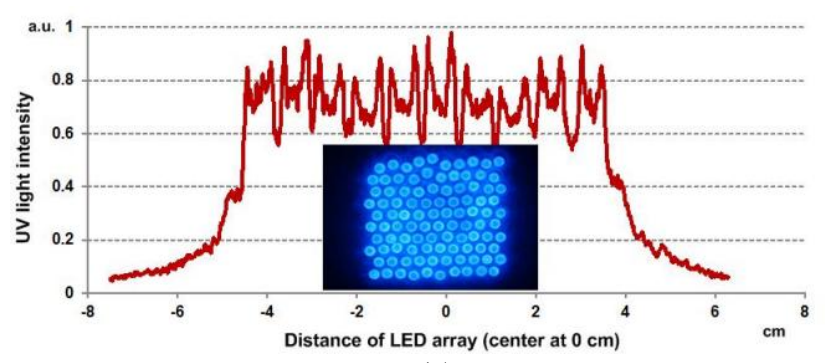

(a)

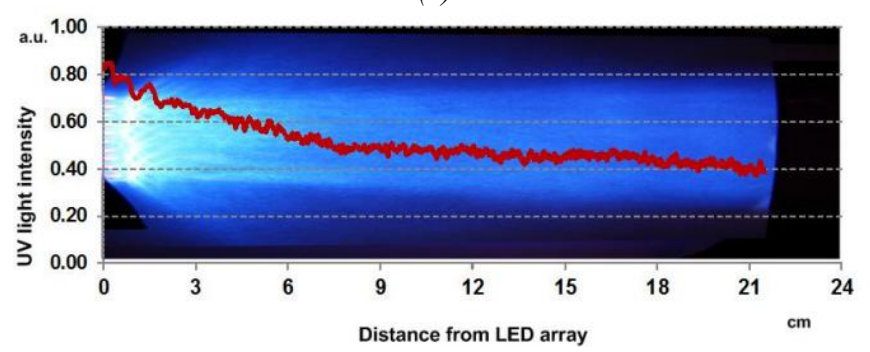

(b)

Figure 4: UV-LED array intensity distribution: (a)Intensity on cross-section view, (b) Intensity attenuation through the distance.

patterned to form LED interconnects. PCB IC sockets were mounted on the PCB to plug in the LEDs instead of directly soldering to the PCB for future possible replacement of LEDs as shown in Figure 3(a). Each LED bulb received the opaque sidewall coating previously discussed. The completed UV light source is shown in Figure 3(b) where the 100 LED array covers an exposure area of 2 by 2 inches.

The areal intensity distribution of the UV-LED array was obtained using the Olympus Wasabi imaging software package [12]. The intensity measurement image of the array is shown in the inset of Figure 4(a), and the intensity of the captured image is plotted as shown in Figure 4(a). The x-axis is the distance in $\mathrm{cm}$ and the $y$-axis is the relative intensity measurement. A reasonable areal uniformity is achieved. The intensity attenuation of the array as a function of distance was also measured and is shown in Figure 4(b). As expected, the intensity decreases as a function of distance. In addition, the intensity distribution as a function of wavelength was measured for the UV-LED light source as well as a conventional UV lithography system (OAI, near UV mercury bulb) with an optical spectrometer (AvaSpec-3648). Figure 5 plots the measured relative intensity from each light source as a function of wavelength.

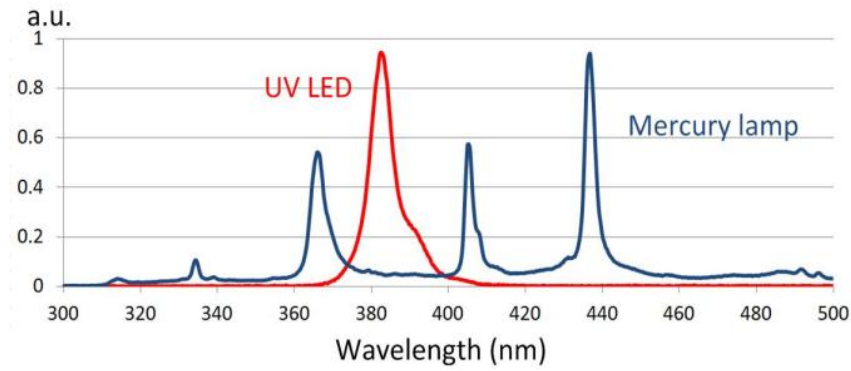

Figure 5: Intensity distribution vs wavelength with UV-LED and conventional mercury lamp. 


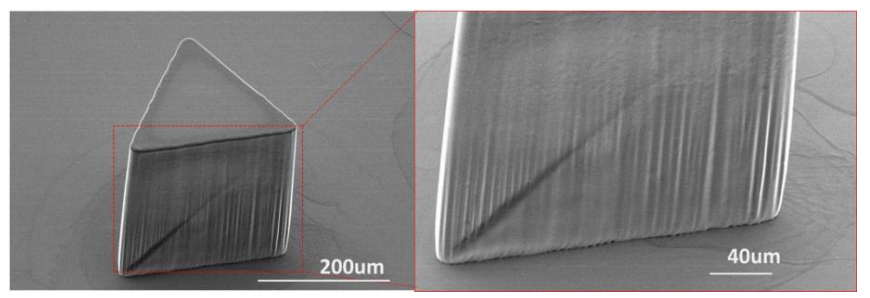

(a)

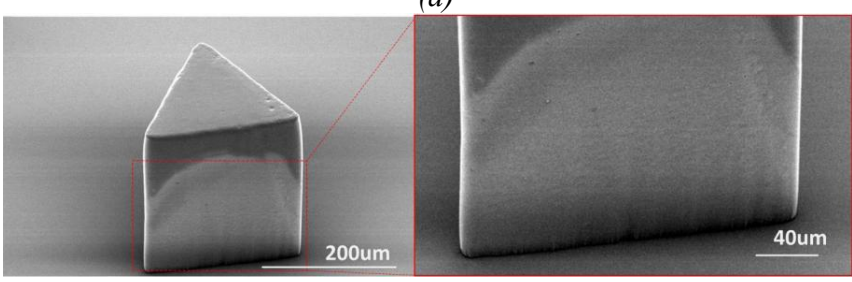

(b)

Figure 6: Comparison of microtriangular pillars fabricated by: (a) mercury lamp exposure scheme (OAI, conventional method), (b) UV-LED lithography scheme.

As expected, the UV-LED source shows a single peak (near-monochromatic) characteristic, while the mercury lamp has typical multiple peaks shown at $365 \mathrm{~nm}, 405 \mathrm{~nm}$ and $436 \mathrm{~nm}$ respectively.

Figure 6 shows a comparison of two triangular columnar SU-8 structures fabricated using a conventional aligner with a mercury lamp source (Figure 6a) and the UV-LED array (Figure 6b). Each was processed under the same soft-bake, post exposure bake, and UV exposure energy (1440 mJ) conditions. As can be seen, both exposure techniques produce structures with comparable features and resolution. To examine this further, and to demonstrate the utility of the UV-LED system, typical patterns in thicknesses of SU-8 widely used in MEMS processing, namely $15 \mathrm{~m}, 50 \mathrm{~m}$, $70 \mathrm{~m}$, and $100 \mathrm{~m}$, have been exposed, developed, and assessed (Figure 7). The light intensity of the UV-LED system was set at

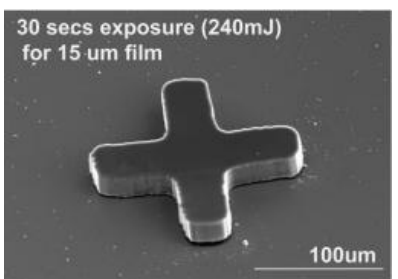

(a)

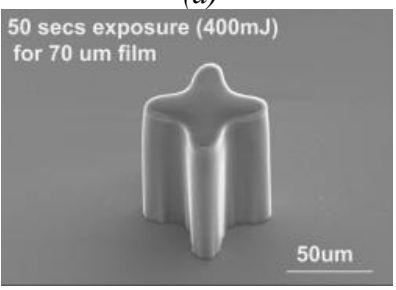

(c)

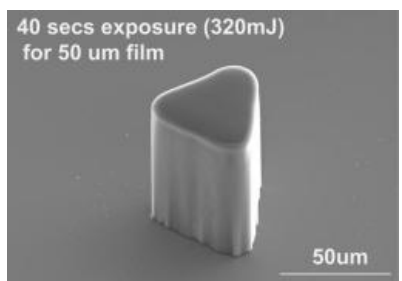

(b)

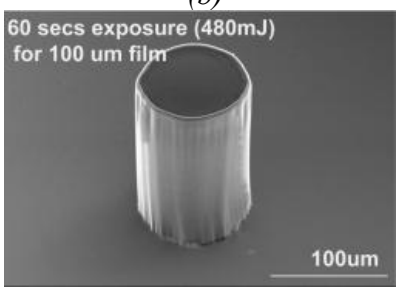

(d)
Figure 7: Microfabricated structures of typical thicknesses of SU-8 by UV-LED lithography (intensity at $8 \mathrm{~mW} / \mathrm{cm}^{2}$ ): (a) $15 \mathrm{~m}$ thick pattern for $240 \mathrm{~mJ} / \mathrm{cm}^{2}$ (30 secs), (b) $50 \mathrm{~m}$ thick pattern for $320 \mathrm{~mJ} / \mathrm{cm}^{2}$ (40 secs), (c) $70 \mathrm{~m}$ thick pattern for $400 \mathrm{~mJ} / \mathrm{cm}^{2}$ (50 secs), (d) $100 \mathrm{~m}$ thick pattern for $480 \mathrm{~mJ} / \mathrm{cm}^{2}$ (60 secs).

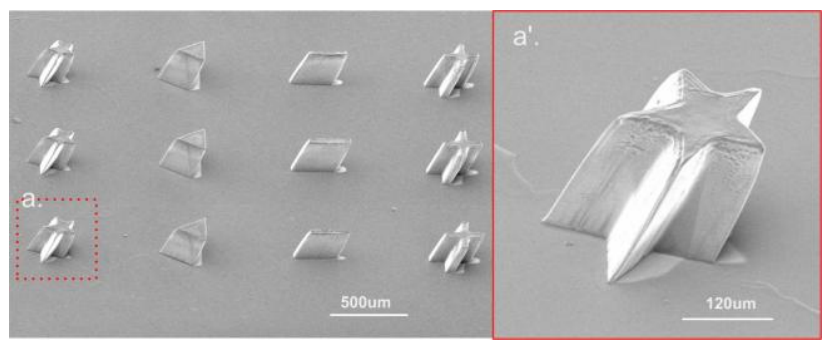

Figure 8: Single static inclined exposure with various geometries; inset ( $(a)$ is the magnified view of (a).

$8 \mathrm{~mW} / \mathrm{cm}^{2}$. The exposure dose of each sample is applied based on the SU-8 datasheet (MicroChem, Inc.), corresponding to $240 \mathrm{~mJ}$, $320 \mathrm{~mJ}, 400 \mathrm{~mJ}$, and $480 \mathrm{~mJ}$ respectively [13]. As can be seen, good reproduction of the patterns is achieved using the UV-LED array system.

\section{ADVANCED UV-LED LITHOGRAPHY METHODS}

One of the potential advantages of the UV-LED system is its ease of use in advanced SU-8 lithography approaches, including multidirectional, rotational, and timed-development-thermal-reflow lithography. Such techniques are being used to create structures in diverse application areas such as microfilters, scaffold structures for cell manipulation, and antenna structures for $\mathrm{GHz}$ and $\mathrm{THz}$ range applications. Previously, modifications to conventional aligners were required to perform these advanced lithographic methods, which may not be desirable in a shared fabrication environment. The UV-LED system is therefore tested in these applications to determine its suitability as a light source for advanced UV lithography.

\section{Multidirectional UV-LED Lithography}

Multidirectional UV lithography is a 3-D microfabrication scheme, based on conventional UV lithography, in which multiple UV exposures with different exposure angles relative to the photomask results in 3-D latent images in the photoresist [5].

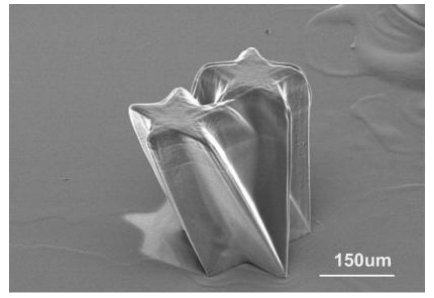

(a)

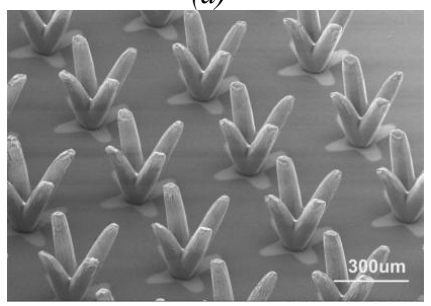

(c)

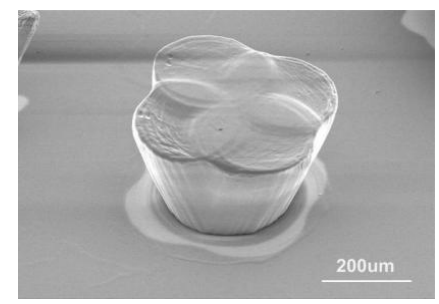

(b)

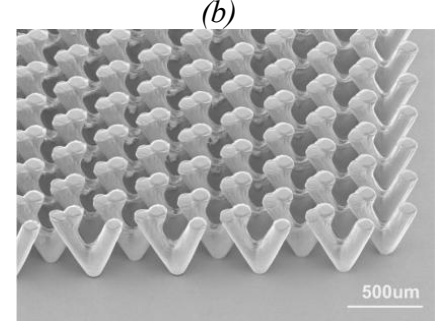

(d)
Figure 9: Multiple static exposure: (a)Micro star shape coupled pillar, (b) Clover horn, (c) Micro cactus field, (d)Anchored micro groove structures. 


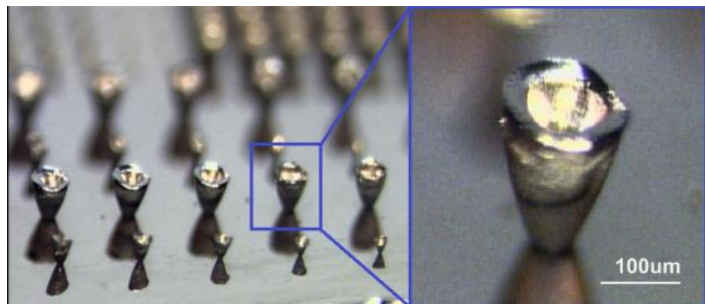

Figure 10: Dynamic mode multidirectional UV-LED lithography for micro 3-D horn array.

A backside exposure scheme is typically utilized, in which the photoresist is coated on a prepatterned $\mathrm{Cr}$ mask to eliminate any air gap between the mask and the thick photoresist. Figure 8 shows various shapes of pillar arrays with $300 \mathrm{~m}$ tall and $40 \mathrm{~m}$ minimum feature size (aspect ratio exceeding 5) and inclined angle of $25^{\circ}$ fabricated from the UV-LED system. $1440 \mathrm{~mJ}$ was applied as an exposure dose which is equivalent to 3 minutes exposure time with UV intensity setting at $8 \mathrm{~mW} / \mathrm{cm}^{2}$. Unlike previous approaches, in which the sample had to be inclined relative to the horizontal exposure tool, the use of the lightweight, compact UV-LED array enabled the tilting of the light source (a $45^{\circ}$ tilt angle) over a horizontally-held substrate. Defining the incident inclined angle of UV light by tilting the UV-LED source eliminates the need of an additional sample stage.

Figure 9 illustrates the multiple-static exposure scheme where multiple UV light exposures are applied with different angles producing complex 3-D microstructures. For example, a four pillar array 'micro cactus field' as shown in Figure 8(c) resulted from a $4 \mathrm{x}$ $\mathrm{UV}$-exposure at a tilt angle of $45^{\circ}$ and a rotation of $90^{\circ}$ between each of the four exposures.

Figure 10 demonstrates a 3-D microhorn array by further utilizing an inclined rotational stage. To expose the horn pattern, the SU-8 coated photomask was rotated with inclined angle of $45^{\circ}$ during the exposure process at a rotational speed of approximately $20 \mathrm{rpm}$.

\section{UV-LED driven timed development and thermal reflow (TDTR) process}

A fabrication process using timed development [7] in the deep via pattern of ultraviolet (UV) lithography with SU-8 and subsequent thermal reflow has been demonstrated to achieve rounded profiles. Concave profile on SU-8 coating was fabricated by time controlled developing on non-exposed area and followed by baking for thermal reflow. Fabricated concave patterns are micromolded by PDMS to produce a convex replica for microlens application as shown in Figure 11.

\section{CONCLUSION}

UV lithography based on an array of UV-LEDs for conventional and three-dimensional exposure of thin and thick photoresists has been developed. The UV-LED approach shows the ability to create structures comparable to those from conventional mercury lamp sources with comparable exposure times. Further, the UV-LED approach has several advantages including simplicity of operation, low power consumption, and reduced system cost. Typical thicknesses of SU-8 resist, namely 15, 50, 70, and $100 \mathrm{~m}$, were successfully patterned. The UV-LED approach was also successfully demonstrated in advanced 3-D lithography, including single and multiple static inclined exposure and dynamic exposure schemes, and timed-development-and-thermal-reflow process.

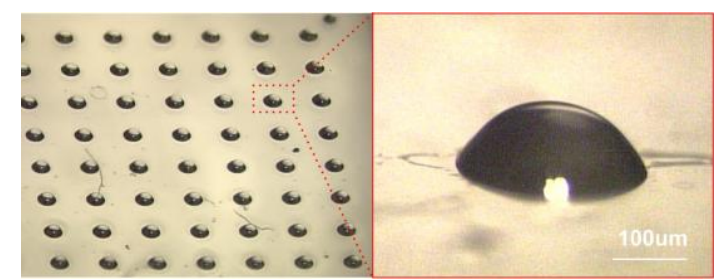

Figure 11: Optical microscopy view of microlens array by TDTR process; inset is the magnified view of a microlens.

Highlighted complex 3-D microstructures such as micro star shape coupled pillar, micro clover horn, micro horn, micro convex lens, micro cactus field, and anchored micro groove structures were successfully fabricated. An advantage of the use of the UV-LED approach in these fabrication schemes is that modification of mask aligners to hold special stages is not required; instead, dedicated equipment for complex lithography can be easily constructed using this light source.

\section{REFERENCES}

[1] Marc J. Madou, Fundamentals of Microfabrication, CRC press, Florida, 2001.

[2] Lin, Burn Jeng, "Deep UV lithography", Journal of Vacuum Science and Technology, Vol 12, Issue 6, (1975), pp1317-1320.

[3] K Jiang, M J Lancaster, I Llamas-Garro, P Jin, "SU-8 Ka-band filter and its microfabrication", J. Micromech. Microeng. 15 (2005) 1522-1526.

[4] Hsiharng Yang, Ching-Kong Chao, Mau-Kuo Wei, Che-Ping Lin, "High fill-factor microlens array mold insert fabrication using a thermal reflow process", $2004 \mathrm{~J}$. Micromech. Microeng. 141197.

[5] Jungkwun 'JK' Kim, Mark G. Allen and Yong-Kyu 'YK' Yoon, "Computer controlled dynamic mode multidirectional UV lithography for 3-D microfabrication," $2011 \mathrm{~J}$. Micromech. Microeng. 21035003.

[6] Hyeon-Don Kim, Gun-Wook Yoon, Jeongho Yeon, Joo-Hyung Lee and Jun-Bo Yoon, "Fabrication of a uniform microlens array over a large area using self-aligned diffuser lithography (SADL)", J. Micromech. Microeng. 22 (2012) 045002 (7pp).

[7] Jungkwun 'JK' Kim, Kangsun Lee, Kwang W. Oh and Yong-Kyu 'YK' Yoon, "Microfabrication of rounded channel and waveguide integrated microlens using timed development and thermal reflow process," uTAS, the 13th international conference, Jeju, Korea, Nov 2009.

[8] Rosanne M. Guijt, Michael C. Breadmore, "Maskless photolithography using UV LEDs", Lab Chip, 2008, 14021404.

[9] S. Suzuki, Y. Matsumoto, "Lithography with UV-LED array for curved surface structure", Microsyst Technol (2008) 14:12911297.

[10] <http://www.superbrightleds.com/moreinfo/component-leds/ 5mm-uv-led-15-degree-viewing-angle-380-nm-30mw/632/> [Accessed: 3 April 2012].

[11] $<$ http://search.newport.com/?q=350\%20watt $\% 20$ mercury $\%$ 20lamp > [Accessed: 3 April 2012].

[12] Hamamatsu Photonics Germany GmbH $<$ www.hamamatsu.com $>$

[13] <http://www.microchem.com/Prod-SU82000.htm> [Accessed: 3 April 2012].

\section{CONTACT}

*Jungkwun Kim: jkim831@gatech.edu 